\title{
Aspergillosis of the nose and paranasal sinuses
}

\author{
C M MILROY, $\dagger$ J D BLANSHARD,* S LUCAS, $\dagger$ L MICHAELS $\dagger$ \\ From the Departments of Histopathology, University College and Middlesex School of Medicine, London, and \\ the * Royal National Throat, Nose, and Ear Hospital, London
}

SUMMARY Fulminant aspergillosis was diagnosed on nasal biopsy in a 49 year old man who had features of an aspergilloma. Further postmortem examination of this area was performed and the results were contrasted with the histological features of other Aspergillus infections. The nasal biopsy specimen and postmortem examination showed infiltrating Aspergillus hyphae with tissue necrosis and little inflammatory response. The hyphae were easily seen with routine stains. This contrasts with the findings in invasive aspergillosis where there is fibrosis and a granulomatous response to the Aspergillus hyphae. The hyphae are seen in giant cells using fungal stains. In the saprophytic infections aspergilloma and allergic Aspergillus sinusitis there is no tissue invasion or destruction. Aspergillus infections of the nose and paranasal sinuses often require biopsy for accurate diagnosis. As treatment varies pathologists need to be able to distinguish the different patterns of infection.

Aspergillosis of the nose and paranasal sinuses is being recognised more and more, and it has been found that four forms of the infection occur each of which has its own distinct histopathological features. Allergic Aspergillus sinusitis and aspergilloma are benign saprophytic infections; invasive aspergillosis is a slowly progressive but destructive infection. Fulminant aspergillosis is a rapidly progressive infection which carries a high mortality and usually affects immunocompromised patients. The histological picture shows infiltrating Aspergillus hyphae with little inflammatory response. We describe here a patient in whom two forms of the disease could be identified concomitantly after a detailed investigation of the paranasal sinuses at necropsy using a special method. As an accurate histological diagnosis of the disease is important for its specific treatment we review the pathological features of the four types of aspergillosis.

\section{Case report}

A 49 year old Nigerian brewery controller was referred from Nigeria with a three month history of pronounced right proptosis, periorbital swelling, and nasal obstruction. He had lost $16 \mathrm{~kg}$ in weight over the preceding six months and had periods of confusion and unsteadiness. He was a non-insulin dependent diabetic, well controlled on chlorpropramide. He had hepatomegaly and a liver biopsy performed three years previously showed cirrhosis, although the exact histology was not known and was unavailable for review. Investigations showed an anaemia of $8.4 \mathrm{~g} / \mathrm{dl}$ Accepted for publication 4 August 1988 with a reticulocyte count of $20 \cdot 2 \%$. Haemolytic anaemia was suspected and investigations suggested a low grade disseminated intravascular coagulopathy. There was no evidence of a haemoglobinopathy. The white cell count was $17.4 \times 10^{6} / 1$ with $66 \%$ neutrophils and $29 \%$ lymphocytes. Liver function tests were abnormal: aspartate aminotransferase $90 \mathrm{IU} / 1$ (normal 9-40), alkaline phosphatase activity $1030 \mathrm{IU} / 1$ (normal 35-105), and gammaglutamyl aminotransferase $957 \mathrm{IU} / 1$ (normal < 45). Albumin concentration was low at $22 \mathrm{~g} / \mathrm{l}$. The prothrombin time was 16 seconds. Serology for human immunodeficiency virus (HIV) 1 and hepatitis B were negative. Chest $x$-ray picture showed apical shadowing in both lungs. Mantoux tests at $1 / 10000$ and $1 / 1000$ were both negative.

\section{Pathology}

Coronal and axial computed tomography scans of the head showed a large mass extending through the walls of the right maxillary antrum and ethmoid sinuses into the orbit (fig 1) and up to the cribriform plate.

At nasoscopy an irregular friable mass was seen to obscure the right nasal airway. A provisional diagnosis of a neoplasm was made and a biopsy performed. This showed nasal mucosa with infiltration by Aspergillus hyphae which were easily recognisable in routine haematoxylin and eosin stained sections. Necrosis was present and there was only a moderate acute inflammatory response. No granulomatous reaction was seen (fig 2). Subsequent culture showed the organism to be Aspergillus fumigatus.

Treatment with amphotericin B was started with 


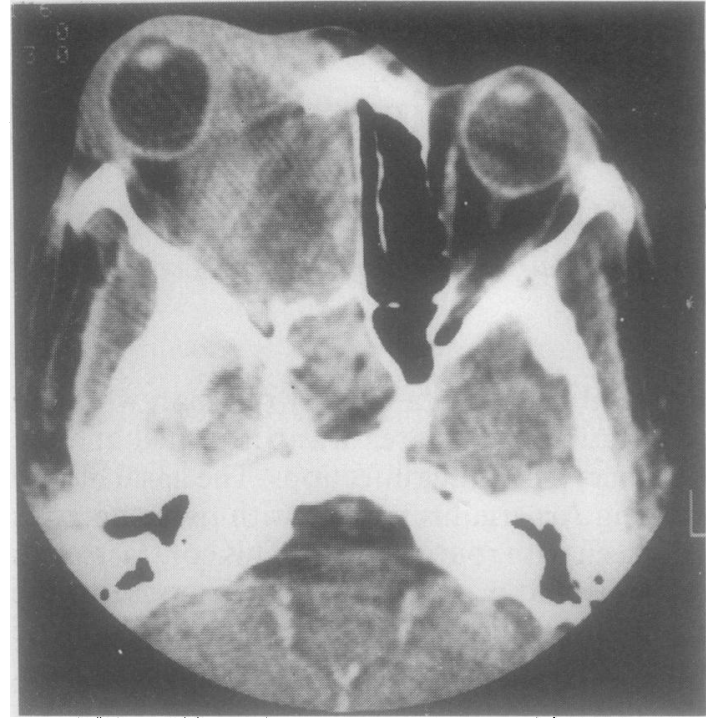

Fig 1 Computed tomography scan of nose and paranasal sinuses showing a mass affecting the right ethmoid and sphenoidal sinuses and orbit.

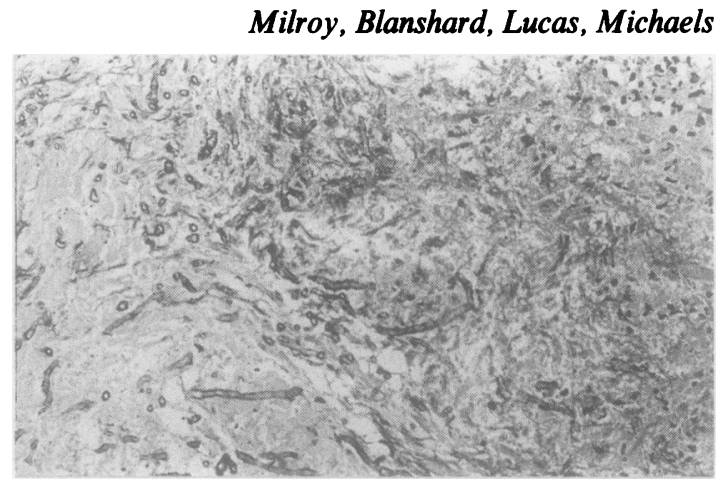

Fig 2 Nasal biopsy specimen showing infiltrating Aspergillus hyphae with necrosis but no granulomatous response. (Haematoxylin and eosin.)

increasing doses, and after further supportive treatment the mass was debulked through a lateral rhinotomy approach. This showed much necrotic material occupying the right maxillary antrum, ethmoid and frontal sinuses, up to and affecting the cribriform plate and penetrating the orbital periosteum into the soft tissues of the orbit. All the vital structures of the orbit

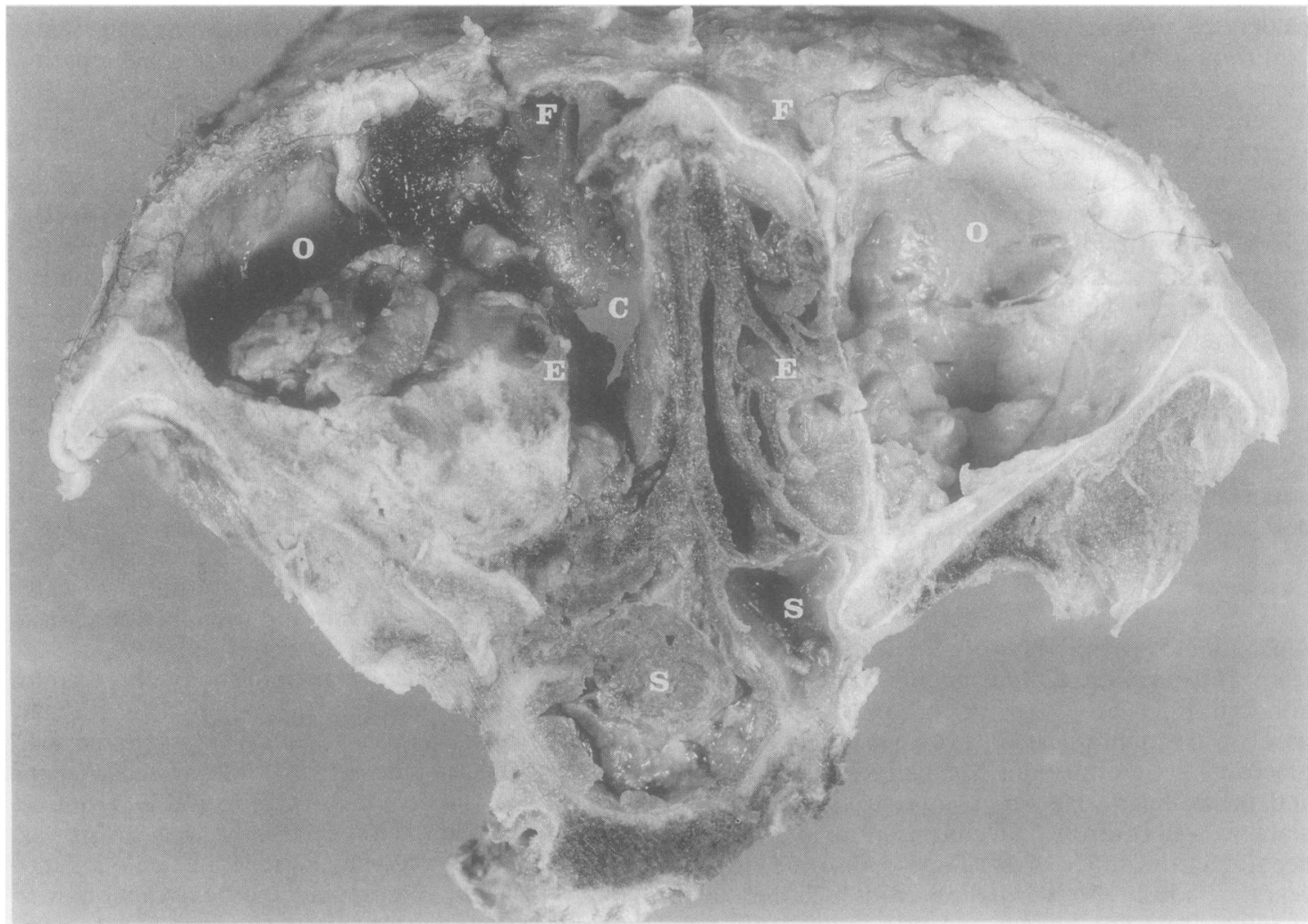

Fig 3 Necropsy specimen of the nose and paranasal sinuses showing Aspergillus in the right ethmoid and sphenoidal sinuses and orbit. Destruction of the cribriform plate is visible. $C=$ Cribriform plate, $E=$ ethmoid sinuses, $F=$ frontal sinus, $O=$ orbit, $S=$ sphenoid sinus. 
were preserved. His further postoperative course was stormy with severe electrolyte and liver function disorder. He died one week later.

At necropsy the nose and paranasal sinuses were removed as a block as described by Michaels. ${ }^{1}$ The block was cut into horizontal slices using a band saw to permit detailed examination of the paranasal sinuses. A necrotic yellow mass was present in the right ethmoid sinus extending into the right orbit. The mass extended up to the cribriform plate which was eroded through to the dura but the frontal lobes of the brain were not affected. Similar material was present in the right frontal and sphenoidal sinuses (fig 3). The right maxillary antrum had been previously operated on but residual necrotic material was present. The fourth upper right and sixth upper left teeth were absent. Histological examination of tissue from the sinus walls showed infiltrating Aspergillus hyphae as seen in the biopsy specimen. The optic nerve and blood vessels had been invaded by Aspergillus hyphae. The centre of the mass in the ethmoid and sphenoidal sinuses showed a solid mass of Aspergillus hyphae which had the appearances of an aspergilloma.

Examination of the empty fourth tooth socket showed infiltrating Aspergillus hyphae extending close to the oral cavity. No Aspergillus hyphae were seen in the frontal lobes or in any other brain tissue, although Aspergillus was observed in association with destruction of the cribriform plate. No granulomatous response to Aspergillus hyphae was seen in any of the material.

Examination of the lungs showed cavitating, fibrosing tuberculosis of both apices from which Mycobacterium tuberculosis was cultured. Histology was typical of tuberculosis with caseating granulomas present with Langhans' giant cells seen. The liver showed a mixed type inactive cirrhosis. Severe occlusive coronary artery atheroma was present. Bone marrow examination showed a reactive marrow. No Aspergillus was seen outside the skull. There was no evidence of renal damage by amphotericin.

\section{Discussion}

Aspergillus is a common saprophyte of soil and decaying organic material such as fruit and other foods. It is a filamentous fungus that has septate hyphae and reproduces as asexual conidia. Specialised fruiting bodies called conidiophores are produced and can be seen in some disease states. Several species have been recognised as causing disease in man but the most common are Aspergillus fumigatus, $A$ flavus, and $A$ Niger. In tissue sections Aspergillus is recognised by the septate hyphae and dichotomous branching at an angle of $45^{\circ}$ (fig 4).

Aspergillus infections of the nose and paranasal

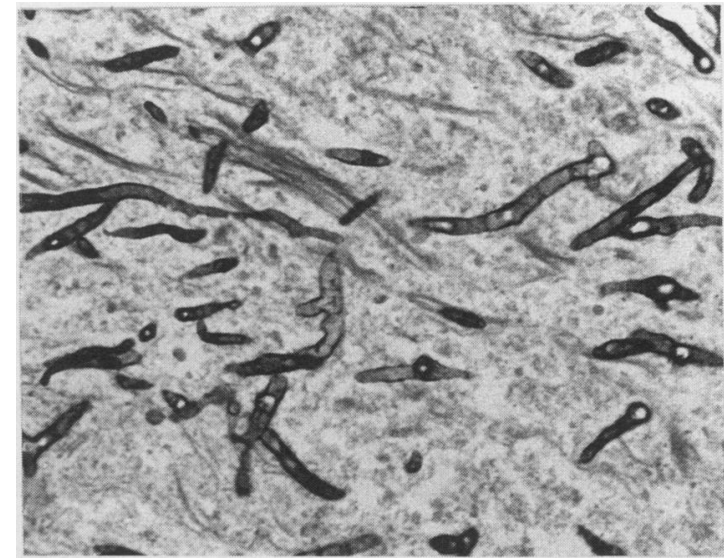

Fig 4 Section of nasal biopsy specimen showing septate Aspergillus hyphae branching at $45^{\circ}$. (Grocott's silver stain.)

sinuses are unusual but have been increasingly recognised in recent years. Fulminant aspergillosis was first recognised in 1980 . The infection is usually described in neutropenic patients with malignant neoplasms of the haemopoietic or lymphoreticular systems which often require bone marrow transplantation. ${ }^{2-4}$ The clinical findings in these patients usually show ulceration of the nasal mucosa, and destruction of the inferior turbinates may be seen. Prominent non-tender facial cutaneous erythema and oedema may be an early manifestation. ${ }^{5}$ The infection will progress with destruction of the sinuses, angio-invasion, and extension into the orbit and brain. There is little tissue reaction and no granulomatous response is seen. Rhinocerebral zygomycosis (mucormycosis) can present in a similar manner and histological differentiation can be difficult as degenerate Aspergillus hyphae have a similar appearance to the broad irregular hyphae of Rhizopus oryzae. In fulminant aspergillosis the hyphae are haematoxyphilic as are the hyphae of Rhizopus oryzae.

The second form of destructive paranasal sinus aspergillosis is termed invasive aspergillosis. ${ }^{6}$ This is a rare disease which presents clinically as an enlarging mass in the cheek, orbit, nose and paranasal sinuses region. Proptosis is often a prominent feature.' A malignant neoplastic process is usually suspected until biopsy confirms the diagnosis. This disease occurs in patients without obvious immune deficiency. There is a granulomatous response to Aspergillus hyphae with considerable fibrosis. The inflammatory process extends beyond the bony walls of the sinuses into the soft tissues of the cheek and orbit. Extension into the brain can occur. ${ }^{8}$ Aspergillus hyphae are scanty and not easily seen with routine stains, appearing as holes in giant cells (fig 5), but can be easily identified with fungal strains such as a silver stain (fig 5 , inset). 


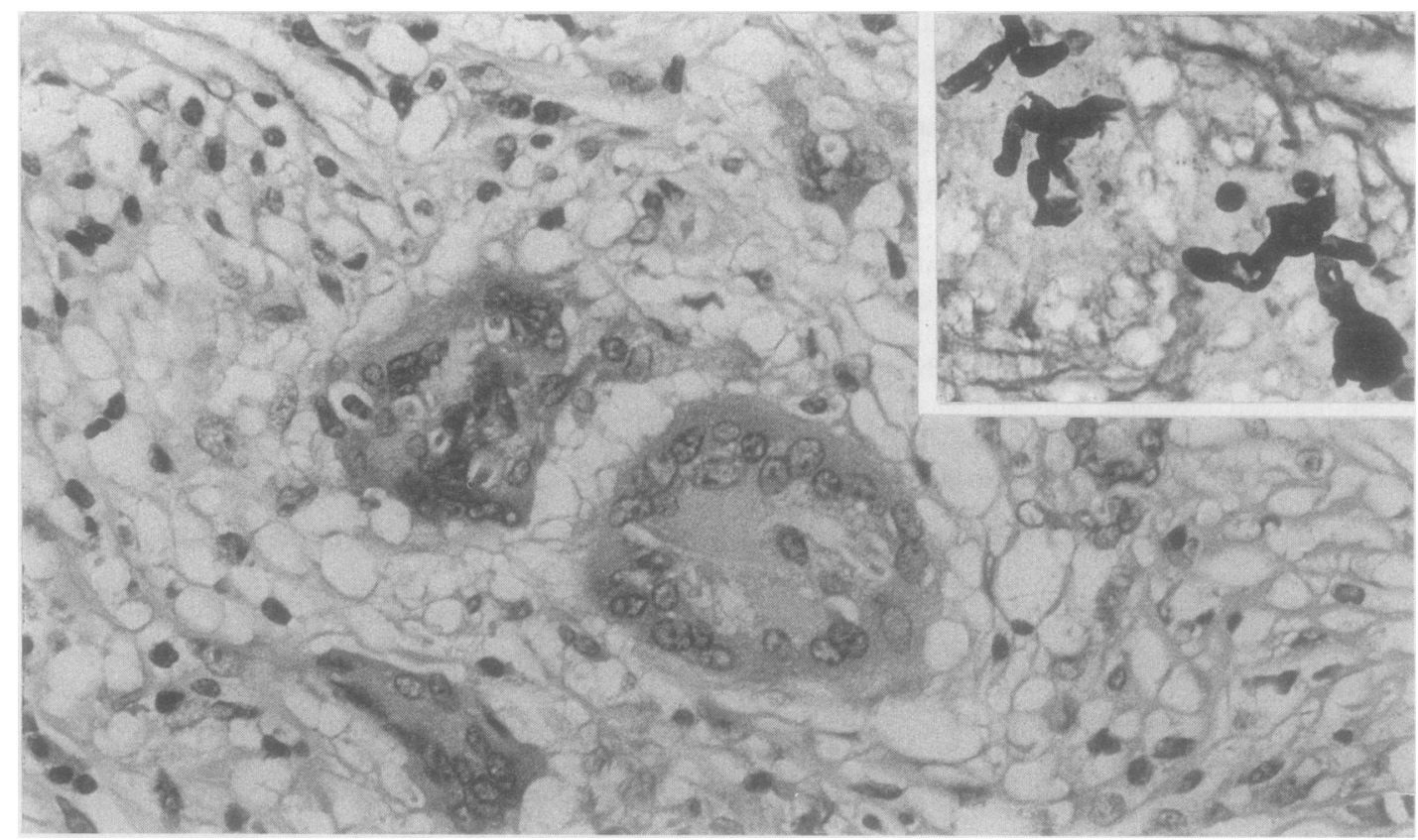

Fig 5 Giant cells in invasive aspergillosis with "holes" of engulfed Aspergillus hyphae. (Haematoxylin and eosin.) Inset: Aspergillus hyphae in giant cells identified by fungal (Grocott's silver) stain.

Management of both fulminant and invasive aspergillosis requires systemic antifungal treatment such as amphotericin B, and surgery is usually required for invasive asperigillosis and sometimes with the fulminant form.

Two saprophytic forms of aspergillosis of the nose and paranasal sinuses are recognised. These are allergic Aspergillus sinusitis and aspergilloma. In aspergilloma a fungus ball of Aspergilli is present in a sinus, usually the maxillary antrum. Histology shows a tangled mycelium of Aspergilli with little inflammatory response. The hyphae are arranged in concentric layers like onion rings. Conidiophores may be seen. The hyphae stain poorly with haematoxylin and eosin. The clinical picture is of a sinus affection and the condition responds well to drainage of the sinus.9

Allergic Aspergillus sinusitis has also been recently recognised. The histological features of this condition are scattered Asperigullus hyphae in pale eosinophilic or basophilic mucin in which sloughed respiratory epithelium, eosinophils, and Charcot-Leyden crystals may be seen. Most patients are young adults with asthma and the disease is analogous to allergic bronchopulmonary aspergillosis. Surgical drainage of the sinuses is often followed by recurrence and corticosteroid treatment has been suggested for this condition. ${ }^{1011}$

Our patient had aspergillosis of the nose and paranasal sinuses which had features of both the fulminant form and of aspergilloma. Ihe exacı immune state of our patient is uncertain. He had a normal granulomatous response to his tuberculosis but had a negative Mantoux test and no granulomatous response to infiltrating Aspergillus hyphae. This response to Aspergillus is usually seen in neutropenic patients. Our patient was not neutropenic but did have cirrhosis, diabetes, and tuberculosis which are known to depress immunity. Although no immune function tests were performed, there is indirect evidence of decreased cell mediated immunity. Data on the immune response to invasive aspergillosis, however, are lacking.

We were interested to note the absence of teeth in this patient. Recent work has suggested that Aspergillus hyphae may gain entry into the maxillary antrum following endodontic treatment of teeth. The material used contains zinc oxide and paraformaldehyde and has been shown to promote the growth of Aspergillus. Even when teeth have been removed that material will remain. ${ }^{1213}$ In our patient Aspergillus hyphae were found in the tooth socket but no zinc oxide material was seen and no other teeth had been treated and it seems unlikely that the Aspergillus gained entry this way.

The microbiological cultures were carried out in the department of microbiology, University College Hospital. 


\section{References}

1 Michaels L. The nose and paranasal sinuses-removal at autopsy. In: Ear, nose and throat histopathology. London: SpringerVerlag, 1987:136-7.

2 McGill TJ, Simpson G, Healy GB. Fulminant aspergillosis of the nose and paranasal sinuses: A new clinical entity. Laryngosope 1980;90:748-54.

3 Landoy Z, Rotstein C, Shedd D. Aspergillosis of the nose and paranasal sinuses in neutropenic patients at an oncology center. Head Neck Surg 1985;8:83-90.

4 Schubert MM, Peterson DE, Meyers JD, Hackman R, Thomas ED. Head and neck aspergillosis in patients undergoing bone marrow transplantation. Cancer 1986;57:1092-6.

5 Weingarten JS, Cockett DM, Lask RP. Fulminant aspergillosis: Early cutaneous manifestations and the disease process in the immunocompromised host. Otolaryngol Head Neck Surg 1987; 97:495-9.

6 Hora JF. Primary aspergilloma of the nose and paranasal sinuses and associated areas. Laryngoscope 1965;75:768-73.

7 Milosev B, Mahgoub ES, Abdel Aal O, El Hassan AM. Primary aspergilloma of the paranasal sinuses in the Sudan. Br J Surg 1969;56:132-7.
8 Lowe J, Bradley J. Cerebral and orbital Aspergillus infection due to invasive aspergillosis of the ethmoid sinus. J Clin Pathol 1986;39:774-8.

9 Michaels L. Infections of the nose and paransal sinuses. In: Ear, nose and throat histopathology. London: Springer-Verlag, 1987: $145-6$.

10 Katzenstein AA, Sale SR, Greenberger PA. Pathologic findings in allergic aspergillus sinusitis. Am J Surg Pathol 1983;7:439-43.

11 Waxman JE, Spector JG, Sale SR, Katzenstein AA. Allergic Aspergillus sinusitis: Concepts in treatment of a new clinical entity. Larngyoscope 1987;97:261-6.

12 Beck-Mannagetta J, Necek D, Grazzerbauer M. Zahnarztliche Aspekte der solitaren Kieferholen-Aspergillose. Z Stomatol 1986;83:283-315.

13 Beck-Mannagetta J, Necek D. Radiological findings in aspergillosis in the maxillary sinus. Oral Surg Oral Med Oral Pathol 1986;62:345-9.

Requests for reprints to: Dr C M Milroy, Department of Histopathology, University College and Middlesex School of Medicine, Institute of Laryngology and Otology, 330/332 Gray's Inn Road, London WCIX 8EE, England. 